\title{
A TERAPIA OCUPACIONAL NO CONTEXTO DA EDUCAÇÃO EM SAÚDE: UM RELATO DE EXPERIÊNCIA
}

\author{
LA TERAPIA OCUPACIONAL EN EL CONTEXTO DE LA EDUCACIÓN \\ PARA LA SALUD: UN INFORME DE EXPERIENCIA
}

\author{
OCCUPATIONAL THERAPY IN THE CONTEXT OF HEATH EDUCATION: AN \\ EXPERIENCE REPORT
}

\author{
Nayana dos Santos FLEXA ${ }^{1}$ \\ Raissa Brígido Ispala CAMARGO ${ }^{2}$ \\ Sara Pacheco NOGUEIRA ${ }^{3}$ \\ Samantha Cristiny Fernandes NUNES ${ }^{4}$
}

RESUMO: A educação em saúde é um método promissor ao enfrentamento das adversidades que afetam a saúde da população. Trata-se de um relato de experiência, com abordagem descritiva. Com objetivo de narrar a experiência vivenciada por estudantes de terapia ocupacional numa Unidade Municipal de Saúde - UMS. Apto e capaz de designar inúmeras formas de cuidado de maneira adequada percebeu-se a terapia ocupacional se sobressai por possuir um olhar apoiador e potencializador. Conclui-se que as atividades confeccionadas apresentaram-se satisfatoriamente favorecendo para a saúde e o bem-estar dos participantes, contudo é importante ressaltar que tais ações devem se adaptadas a rotina das unidades.

PALAVRAS-CHAVE: Atenção primaria á saúde. Educação em saúde. Terapia ocupacional.

RESUMEN: La educación en salud es un método prometedor al enfrentamiento de la adversidad que afectan la salud de la población. Se trata de un informe de experiencia, com enfoque descriptivo. Con el objetivo de narrar la experiencia vivenciada por las estudiantes de terapia ocupacional en una Unidad Municipal de Salud - UMS. Apto y capaz de designar muchas maneras de atención adecuadamente, se dio cuenta de que la terapia ocupacional sobresale por tener una mirada de apoyo y potenciación. Se concluyó que las actividades hechas se han presentado satisfactoriamente favoreciendo la salud y el bienestar de los participantes, es importante comprender sin embargo que tales acciones deben adaptarse a la rutina de las unidades.

PALABRAS CLAVE: Atención primaria de salud. Educación en salud. Terapia ocupacional.

${ }^{1}$ Universidade da Amazônia (UNAMA), Belém - PA - Brasil. Graduação em Terapia Ocupacional. ORCID: https://orcid.org/0000-0003-1423-1742.E-mail: nayannaflexa42@gmail.com

${ }^{2}$ Universidade da Amazônia (UNAMA), Belém - PA - Brasil. Graduação em Terapia Ocupacional. ORCID: https://orcid.org/0000-0003-2624-0734.E-mail: rabrigido.ic@gmail.com

${ }^{3}$ Universidade da Amazônia (UNAMA), Belém - PA - Brasil. Graduação em Terapia Ocupacional. ORCID: https://orcid.org/0000-0002-3089-8945. E-mail: sarapnogueira7@gmail.com

${ }^{4}$ Universidade da Amazônia (UNAMA), Belém - PA - Brasil. Graduação em Terapia Ocupacional. ORCID: https://orcid.org/0000-0002-6363-7741.E-mail: samanthafnunes@hotmail.com 
ABSTRACT: Health education is a promising method to cope with adversities that affect the health of the population. This is an report of experience, with descriptive approach Aiming to narrate the experience experienced by students of occupational therapy in a municipal health unit - UMS. Apt and able to designate numerous forms of care in an adequate way one perceived occupational therapy if it stands out for having a supportive and enhancing look. It is concluded that the manufactured activities presented themselves satisfactorily favoring the health and well-being of the participants, however it is important to emphasize that such actions should adapt to the routine of the units.

KEYWORDS: Primary attention to health. Health education. Occupational therapy.

\section{Introdução}

Nelson Mandela certa vez declarou que "a educação é a arma mais poderosa que pode ser utilizada para mudar o mundo”. Frase essa que expressa o importante papel da educação como instrumento de mudança na sociedade. Diante disso, para que se impacte positivamente e assim fazer a diferença na vida das pessoas, a saúde deve começar pela educação (PITAGUARI, 2016).

Explana Helman (1994) que a maioria da sociedade padece de algum desconforto físico ou emocional e desfrutam de inúmeras autoajudas ou buscam ajuda de outros. Silva e Seffner (2015) complementam mencionando alguns cuidados, como por exemplo: tomar um chá; procurar um médico, curandeiro ou benzedeira; recorrer às crenças ou um familiar, amigo, vizinho; a utilização de terapias energéticas, como: Reiki, Aromaterapia, Acupuntura, Massoterapia, entre outras. Importando-se assim, apenas na eficácia imediata em aliviar a sensação de desconforto das pessoas que buscam tratamento.

Os feitos e as atribulações as quais topamos hoje no âmbito da saúde são reflexos de uma circunstância histórica e político-social que o Brasil confrontou ao longo do tempo (POLIGNANO, 2005). No Brasil a partir do século XIX, que se tiveram as primordiais propostas de educação em saúde (REIS et al. 2013).

Ainda de acordo com o autor, tais ações encontravam-se voltadas às condutas de higiene, já que se visava à introdução e aos costumes europeus na elite brasileira. Restando aos demais, as medidas de cunho sanitarista campanhista, na qual iniciaram só no final daquele século, possuía o intuito do controle de algumas epidemias, como por exemplo: a febre amarela, que causava danos ao comércio.

Declama Salci et al. (2013) como multifacetada a educação em saúde, devido às suas inúmeras proporções, como: políticas, filosóficas, sociais, religiosas, culturais, além de incluir aos aspectos práticos e teóricos do indivíduo, grupo, comunidade ou sociedade. Em duas 
facetas engloba-se o processo saúde-doença, se fazendo necessária para a preservação ou para evitar ou retardar a presença de doenças e faz-se essencial para trazer qualidade de vida à pessoa ou retardar as complicações do processo ao adoecimento.

Neste ínterim, um profissional da saúde que se encaixa entre educação e saúde é o Terapeuta Ocupacional, visto que se entrega como agente impulsionador da educação em saúde, pois dedica-se na busca por compreender as relações saúde-sociedade (ROCHA; SILVA; RAIMUN-DO, 2016). Complementa Rocha e Souza (2011), que, embora o aparecimento no Brasil da profissão em Unidades Básicas de Saúde - UBS tenha desenrolado desde a década de 90 em cidades desiguais, como: São Paulo, Belo Horizonte, Camaragibe e Sobral, constata-se a evidência por Sousa (2015) que tais profissionais estão capacitados e habilitados a serem contratados. Sendo assim possível, sua introdução ser aos serviços em geral de Saúde, isto é aos dispositivos de Atenção Primária a Saúde - APS, como: as Unidades de Saúde da Família - USF e as equipes no qual constituem o serviço, como: o Núcleo Ampliado de Saúde da Família e Atenção Básica - NASF-AB, Consultório na Rua CnR e equipes de Saúde da Família - eSF, nos serviços das Rede de Atenção Psicossocial RAPS, como: aos diversos tipos de Centros de Atenção Psicossocial - CAPS, o Pronto Atendimento em Saúde Mental - PASM, a Unidade de Atendimento Infantil - UAI e o Serviço Residencial Terapêutico (SRT) - ou Residência Terapêutica.

Com isso, o presente estudo teve como objetivo narrar a experiência vivenciada por estudantes da Terapia Ocupacional em época de prática supervisionada numa Unidade Municipal de Saúde - UMS.

\section{Procedimentos Metodológicos}

Este estudo tem abordagem descritiva e caracteriza-se como um relato de experiência resultante a observação por discentes em época de Atividade Prática Supervisionada - APS do Curso de Terapia Ocupacional da Universidade da Amazônia - UNAMA, a disciplina vinculada foi a Terapia Ocupacional em Geriatria e Gerontologia.

Em período de 30 de out. á 04 de dez. de 2017 uma vez por semana no turno da manhã, as ações educativas foram realizadas numa Unidade Municipal de Saúde - UMS situada no município de Ananindeua no Estado do Pará-PA, localizada no norte brasileiro, o público alvo da ação foram adultos e idosos e seus acompanhantes.

Durante as ações foram utilizados recursos de baixo custo confeccionado pelas discentes, a fim de promover uma maior dinamicidade ao aprendizado e de uma linguagem 
acessível, pois conforme Brasil (2007), as dinâmicas e brincadeiras revelam-se serem técnicas afetivo-participativas na educação em saúde. A partir das requisições observadas na própria UMS, as temáticas propostas foram envolvendo: ao Estresse; a Diabetes e Hipertensão e a Saúde do Homem.

Autorizadas pela unidade de saúde, com a premissa de que as mesmas estivessem supervisionadas e que nenhuma informação sigilosa fosse revelada ou comprometesse a identidade dos participantes e a unidade onde os encontros foram realizados.

\section{Resultados e discussão}

A introdução na prática a todo o momento nos encaminha a refletir, como e onde vamos pôr com lucidez e clareza a aprendizagem adquirida na academia. Visto que tal processo esse requer por tantas vezes um levantamento momentâneo baseado nas áreas pela qual vamos atuar, nesse caso a Atenção Básica de Saúde - ABS, a qual envolve uma clientela que vai da criança ao idoso, nas suas diferenciadas problemáticas.

O primeiro encontro no local com a equipe nós deixou bem à vontade para conduzir as ações que iríamos utilizar, mas é importante comentar que enfrentamos outro desafio, que foi trabalhar em equipe e a rotina agitada de uma unidade. A primeira temática trabalhada foi voltada a esclarecer dúvidas e fornecer informação aos usuários da unidade acerca da Terapia Ocupacional, visto que o serviço foi implantado na unidade por meio da prática acadêmica, em primeiro momento notou-se que muitos dos usuários apresentavam-se receosos, mas, ao mesmo tempo com interesse de compreender melhor o trabalho desenvolvido pela profíssão do Terapeuta Ocupacional.

No segundo encontro houve-se a segunda temática voltada ao Estresse no cotidiano, através da aplicação da Escala de Percepção de Estresse-10 (Eps-10). Aqueles que participam e obtiveram escores medianos ou altos de estresse, foi the orientado a procurar por um profissional e previamente lhe foi explicado os impactos causados no cotidiano e, alternativas de redução, como por exemplo: o uso de práticas integrativas e complementares, a inserção de atividades de lazer; mudança de hábitos, entre outros.

No terceiro encontro as ações voltaram-se para os hipertensos e diabéticos da unidade, patologias essas que causam preocupação aos profissionais da saúde. Medina (2019) informa que a diabetes expõe-se a inúmeras complicações, a neuropatia (lesão dos nervos) sendo uma delas, capaz de designar sintomas como adormecimento ou dor, provocando alterações da função motora e da sensibilidade. 
A terceira temática denominada "Diabetes e a sensibilidade" se deu por meio de folders educativos com intuito de prevenir a neuropatia, controle da diabetes e um estilo de vida saudável (alimentação, atividade física, evitar bebidas alcoólicas, fumo etc.). Utilizou-se um pé confeccionado pelas discentes de garrafa pet que didaticamente ajudou na orientação sobre atenção e cuidados aos pés.

Levando um alerta sobre a importância do controle da taxa glicêmica (açúcar no sangue) com intenção de evitá-las no futuro (MEDINA, 2019). Se houve o quarto encontro com a temática denominada "Complicações da Diabetes", em forma de dinâmica usando placas de verdadeiro e falso também confeccionado pelas discentes, para minimizar o impacto do assunto.

A Terapia Ocupacional se destaca pelo uso da atividade humana como instrumento de trabalho. Segundo Silva e Emmel (1993) toda atividade e examinada e avaliada passando por uma criteriosa análise, a fim de distinguir a cooperação na qual tem para o paciente. Através de tal análise, podem ser detectadas habilidades motoras, sensoriais, cognitivas, emocionais e sociais envoltas com a atividade que se pretende utilizar com o paciente.

O quinto e último encontro voltou-se a população masculina de abrangência na unidade, com o início do mês de novembro houve-se a incentiva de conversar com o público, sobre a importância de cuidar da saúde. Dado isso, houve a quinta temática nomeada "Homem também se cuida" através de folder educativo promover a informação sobre exames que avaliam a próstata em especial o do toque renal, com o intuito de vencer o preconceito.

A equipe da UMS iria promover uma ação em saúde em um centro comunitário e fomos convidadas a participar e conduzir uma palestra e atividades para os usuários participantes, e assim tivemos a possibilidade de compartilhar espaços de atuação com médicos, enfermeiros, assistentes sociais entre outros profissionais, e conseguimos contribuir levando um pouco da visão da Terapia Ocupacional para os mesmos e aos usuários.

A vivência prática com adultos e idosos além de prazerosa foi de grande aprendizado. Houve-se a oportunidade da realização de oficinas, dinâmicas e muitas conversas acolhedoras fez-se notar através delas a interação entre si dos usuários participantes em benefício de sana as dúvidas e incômodos. Entretanto observou-se que alguns apresentaram um conhecimento considerado insuficiente sobre o assunto, e demonstraram insatisfação com o serviço público.

Contudo foi notória a preocupação dos mesmos em busca de informação, principalmente com referência as comorbidades patogênicas, como: Hipertensão e Diabetes. Assim, esta experiência mostrou-se uma importante ferramenta para motivar a comunidade em 
questões relacionadas à promoção da saúde em busca da autonomia, da valorização do autocuidado e do desenvolvimento de habilidades.

Assim como, é importante ressaltar o trabalho na comunidade, Souza; Colomé; Costa e Oliveira (2005) evidenciam em seus estudos que tal trabalho oportuniza aos profissionais de saúde inteirar-se na realidade e potencialidades do meio, com o fim de estimular aos participantes a obter estratégias coletivas ao embate dos problemas vividos por essa comunidade.

Ainda de acordo com os autores, o indivíduo ao envolver-se em grupo, tem a oportunidade de expor seus pensamentos, em dar sua opinião, seu ponto de vista, ou seu silêncio, beneficiando-se por meio das trocas de experiências que ocorrem entre eles e, através das contribuições dos profissionais de saúde. Mesmo diante imprevisto e difículdades pequenas até, foi uma prática positiva e agregadora tanto na vida profissional quanto no pessoal. Assim, vamo-nos dando conta de o quanto é importante a função de um Terapeuta Ocupacional em locais que ainda não o tem inserido em sua equipe.

\section{Considerações finais}

Fica evidente o quanto o Terapeuta Ocupacional contribuiu para melhorar a qualidade de vida dos usuários do sistema básico de saúde, exercendo ações positivas na prevenção e promoção da saúde das pessoas nas Unidades Básicas de Saúde, gerando o aperfeiçoamento pessoal e profissional na assistência à Saúde, na Educação e na Assistência Social da profissão.

Grande parte dos estudos encontrados foi de publicação em periódicos de Enfermagem, na qual se mostrou empenhada em debater a temática, porém poucos estudos em outras áreas publicados existem em pequena minoria em especial da Terapia Ocupacional, um profissional da saúde que se enquadra nessa configuração de desenvolvimento entre educação e saúde.

Ante todo o exposto, um fator importante a se destacar é que a educação e a saúde estão interligadas, dessa forma, compreende-se que o profissional de saúde prática educação em saúde em seu cotidiano profissional sem que se perceba como tal. Diante disso, é possível ressaltar a relevância da iniciativa do profissional de saúde trabalhar com a promoção da saúde, seja na área da saúde, social ou até educacional.

São inúmeros os desafios que os profissionais de saúde enfrentam diariamente em função das condições precárias do sistema de saúde no Brasil. Contudo, a educação em saúde 
apesar de que possua métodos e segmentos distintos, não se limita apenas em disseminar conhecimento à comunidade, constitui também em vínculos entre assistidos e profissionais, para assim promover a participação ativa da comunidade, quanto a hábitos que pactuem a saúde e qualidade de vida daquela população.

Conclui-se que, algo a qual deve ser posto em consideração que tais ações devem se adaptadas a rotina da UMS, para assim poder funcionar adequadamente e ativamente, pra no fim trazer bons resultados. Assim como a pouquidade de treinamento dos profissionais de saúde, as quais copiosamente replicam grupos e atividades já existentes nas UMS, sem repensar numa reestruturação ou repaginação dos mesmos, para que assim melhorem suas práticas em ações coletivas, principalmente em grupos de educação em saúde.

\section{REFERÊNCIAS}

BRASIL. Ministério da Saúde. Secretaria de Gestão Estratégica e Participativa. Departamento de Apoio à Gestão Participativa. Caderno de educação popular em saúde. Brasília, DF: Ministério da Saúde, 2007.

HELMAN, C. G. Cultura, saúde e doença. Trad. Eliane Mussnich. 2. ed. Porto Alegre, RS: Artes Médicas, 1994. 333 p.

MEDINA, J. L. Tipos de neuropatia diabética. 2019. Disponível em: https://apdp.pt/viverbem-a-diabetes/tipos-de-neuropatia-diabetica/. Acesso em: 15 nov. 2020.

PITAGUARI, A. Educação: base de saúde e bem-estar? 2016. Disponível: http://autonomiaemsaude.net.br/. Acesso em: 15 nov. 2020.

POLIGNANO, M. V. HISTÓRIAS DAS POLÍTICAS DE SAÚDE NO BRASIL. Uma pequena revisão. 2005. Disponível em: http://medicinadeemergencia.org/. Acesso em: 31 mar. 2021.

REIS, T. C. et al. Educação em saúde: aspectos históricos no Brasil. J Health Sci Inst., v. 31, n. 2, p. 219-223, 2013.

ROCHA, E. F.; SOUZA, C. C. B. X. Terapia Ocupacional em reabilitação na Atenção Primária à Saúde: possibilidades e desafios. Revista de Terapia Ocupacional da Universidade de São Paulo, v. 22, n. 1, p. 36-44, jan./abr. 2011. DOI: https://doi.org/10.11606/issn.2238-6149.v22i1p36-44

ROCHA, P. S; SILVA, S. S; RAIMUNDO, J. B. Educação em saúde no âmbito escolar: ações da terapia ocupacional. Revista Criar Educação, 2016. DOI:

http://dx.doi.org/10.18616/ce.v0i0.2918

SALCI, M. A. et al. Educação em saúde e suas perspectivas teóricas: algumas reflexões. Texto Contexto Enferm., Florianópolis, v. 22, n. 1, p. 224-300, jan./mar. 2013. 
SILVA, A. D.; SEFFNER, F. Educação em saúde: o que se ensina e o que se aprende? Representações de saúde e doença para homens idosos em situação de adoecimento. Textura, n. 33, jan./abr. 2015.

SILVA, C. C. B.; EMMEL, M. L. G. Jogos e brincadeiras: roteiro de análise de atividades para o terapeuta ocupacional. Cadernos de Terapia Ocupacional da UFSCar, São Carlos, v. 4 , n. 1 , p. 46-62, 1993.

SOUSA, E. N. Q. Inserção do terapeuta ocupacional em dispositivos da Rede de Saúde no município de João Pessoa. 2015. 60 f. Monografia (Trabalho de Conclusão do Curso de Terapia Ocupacional) - Universidade Federal da Paraíba, João Pessoa, 2015.

SOUZA, A. C.; COLOMÉ, I. C. S.; COSTA, L. E. D.; OLIVEIRA, D. L. L. C. A Educação em saúde com grupos na comunidade: uma estratégia facilitadora da promoção da saúde. Rev Gaúcha Enferm., Porto Alegre, v. 26, n. 2, p. 147-530, ago. 2005.

\section{Como referenciar este artigo}

FLEXA N. S.; CAMARGO R. B. I.; NOGUEIRA S. P.; NUNES S. C. F. A Terapia Ocupacional no contexto da educação em saúde: um relato de experiência. Temas em Educ. e Saúde, Araraquara, v. 17, n. 00, p. e021007, 2021. e-ISSN 2526-3471. DOI: https://doi.org/10.26673/tes.v17i00.14925

Submetido em: 23/03/2021

Revisões requeridas: $26 / 03 / 2021$

Aceito em: 01/04/2021

Publicado em: 20/04/2021 